\title{
Relación de complementariedad entre los documentos de los archivos y las bibliotecas
}

\author{
Miguel Ángel Rendón Rojas *
}

\section{Artículo recibido: \\ 12 de noviembre de 2020 \\ Artículo aceptado: \\ 10 de marzo de 2021}

Artículo de investigación

\section{Resumen}

Según la Archivística y la Bibliotecología existe una clara diferencia entre fondo y colección. Mientras que fondo es el conjunto de documentos derivados de las actividades de una persona o institución en e1 transcurso de su gestión, por lo que su origen se considera un proceso natural, es un "todo orgánico" y es objeto de atención de la Archivística, la colección es su opuesto, porque su origen es subjetivo y arbitrario, y no puede ser tratada con métodos archivísticos, sino bibliotecológicos. Sin embargo, en ocasiones fondos y colecciones coexisten en archivos y bibliotecas y deben ser gestionados indistintamente por archivistas y

* Instituto de Investigaciones Bibliotecológicas y de la Información, UNAM, México marr@unam.mx INVESTIGACIÓN BIBLIOTECOLÓGICA, vol. 35, núm. 88, julio/septiembre, 2021, México,
ISSN: 2448-8321 pp. 63-82 
bibliotecólogos, aunque teóricamente no les competería. El objetivo del presente artículo es brindar un modelo teórico para explicar esa anomalía. El resultado obtenido es que la relación de complementariedad entre conceptos sirve como modelo explicativo de esa aparente incoherencia. El estudio se fundamenta con la Archivística, la Bibliotecología, la Lógica y la Teoría de Conjuntos. Los métodos utilizados son el hermenéutico, el de análisis y el deductivo. Como corolario se propone la necesidad de realizar un estudio epistemológico más profundo, revisar el objeto de estudio de las mencionadas disciplinas y su interrelación que conduce a la transdisciplina.

Palabras clave: Archivística; Fondo; Colección; Relación entre Conceptos

The relationship of complementarity between documents in archives and libraries

Miguel Ángel Rendón Rojas

\section{Abstract}

According to Archival Science and Library Science there is a clear difference between fonds and collection. While the fonds is the set of documents derived from the activities of a person or institution in the course of its management, its origin is considered a natural process, it is an "organic whole" and is the object of attention of archival science; the collection is opposite to the fonds, because its origin is subjective and arbitrary, so it cannot be treated with archival methods, but with librarianship methods. However, sometimes fonds and collections coexist in archives and libraries and should be managed indistinctly by archivists and librarians, although theoretically it is not their responsibility. The objective of this article is to provide a theoretical model to explain this anomaly. The result obtained is that the relationship of complementarity between concepts serves as an explanatory model for this apparent incoherence. The study is based on Archival Science, Library Science, Logic and Set Theory. The methods used are hermeneutic, analytical and deductive. As a corollary, it is proposed the need to carry out a deeper 
epistemological study, to review the object of study of the mentioned disciplines and their interrelation, wich leads to transdiscipline.

Keywords: Archival Science; Fonds; Collection; Relationship between Concepts

\section{INTRODUCCIÓN}

Si bien se presentan avances dentro de las teorías de las disciplinas informativo-documentales -Archivística, Bibliotecología, Ciencia de la Información, Documentación, Museología- como, por ejemplo, las propuestas de la escuela canadiense en Archivística (Tognoli, 2010), la inclusión de los estudios de la información dentro de la Bibliotecología, o la inserción del factor social en la Ciencia de la Información (Hjørland, 2004), lo que lleva a reconocer más ampliamente la interdisciplina o incluso la transdisciplina entre ellas, aún es generalizada la visión de la autonomía de cada una de esas disciplinas y de las instituciones que son objeto de su atención: archivos, bibliotecas, centros de documentación o museos. Aunque estén físicamente juntas, se distinguen claramente entre sí. Entre las diferencias específicas de cada institución informativa documental encontramos el tipo de documentos con que trabajan y las metodologías propias para su tratamiento.

No obstante esa inconmensurabilidad, en ocasiones, en una misma unidad de información, concretamente en un archivo o en una biblioteca, coexisten conjuntos de documentos de naturaleza heterogénea (fondos versus colecciones), y deben recibir un tratamiento documentario que no correspondería realizar stricto sensu a un profesional que fuera consecuente con su visión de su disciplina: un archivista no debería organizar una colección; o un bibliotecario, un fondo. Sin embargo, en la práctica se hace a un lado la ortodoxia y se llevan a cabo esas actividades. La explicación a esa incoherencia se encuentra en el nivel pragmático; en el caso del archivista, ciertamente no le compete realizar ese tipo de trabajo, pero se hace "Como procedimiento de excepción [...] cuya justificación no tendría que buscarse en la teoría archivística y sí en la necesidad de aplicar un criterio de orden [...] que permita el uso de la documentación" (Torres Monroy, 2000: 38. Resaltado propio). Pero no podemos quedarnos en ese nivel, sino pasar a lo teórico o incluso metateórico, por lo que es necesario analizar conceptos o teorías de las ciencias implicadas. Por ejemplo, aclarar el contenido de los conceptos 
"colección" y "fondo", "archivo sonoro" o "fonoteca, "archivo fotográfico" o "fototeca", entre otros pares similares. Asimismo, se deben resolver problemas sobre el objeto de estudio de la Archivística y de la Bibliotecología, las interrelaciones entre ellas; es decir, resolver el problema crítico del conocimiento planteado por Kant (2007: 8) el cual, aplicado a esas disciplinas informativo-documentales, toma la forma de decidir la posibilidad o imposibilidad de la Archivística y la Bibliotecología como ciencias, de elucidar sus fuentes, extensiones y límites. El hecho de que autores reconocidos en el área archivística dedicaron un apartado al análisis de esa anomalía muestra su importancia: Cruz Mundet (1994: 70-74), Lodolini (1993: 257-272), Heredia (1991: 156-170), Schellenberg (1987: 47-57), Tanodi (2009: 76-91).

Metodología. La pregunta que se pretende responder en el presente estudio es ¿cómo explicar, utilizando una herramienta teórica y no solamente la necesidad práctica, la coexistencia de conjuntos documentales disímiles -colecciones y fondos- en archivos y bibliotecas, situación que desde la teoría de cada disciplina -Archivística y Bibliotecología- no debería tener lugar? Por consiguiente, el objetivo de este artículo es proporcionar una herramienta teórica que permita comprender esa discordancia. La hipótesis que planteamos consiste en que la relación de complementariedad entre conceptos explica esa situación problemática. Como hipótesis complementaria proponemos que es necesario revisar el objeto de estudio de las mencionadas disciplinas y su interrelación. Las herramientas teóricas que utilizamos son las teorías archivística y bibliotecológica para definir el concepto de fondo y colección, así como el tratamiento que se les realiza a cada uno de esos conjuntos documentales. Asimismo, recurrimos a la teoría del concepto propia de la Lógica para determinar las relaciones que existen entre esas formas del pensamiento, y dentro de esas relaciones nos centramos en la de complementariedad. Finalmente, también empleamos la Teoría de Conjuntos para entender esas relaciones y representarlas con ayuda de diagramas de Venn. La metodología empleada incluye el uso del método hermenéutico que nos ayuda a la comprensión de las ideas de los autores consultados a través de la interpretación de sus textos, develando su sentido y significado. La hermenéutica que seguimos es la de Gadamer (1997), para quien la construcción de sentidos se realiza dentro del “juego hermenéutico", donde no ocurre pura creación o invención de significados, ni tampoco mera repetición de éstos. La interpretación se realiza a partir de tradiciones de interpretación (contexto personal, cultural, lingüístico, cognitivo, social, histórico, etc.) que forman el "horizonte hermenéutico". Por consiguiente, mi interpretación está dirigida por el campo de estudio, el problema a resolver y el marco teórico que se utiliza. Otro método al que recurrimos en nuestro estudio es el de análisis para descomponer en sus elementos 
mínimos las propuestas e ideas consultadas, los presupuestos de donde parten, las relaciones internas que presentan y consecuencias a las que conducen. Finalmente, para obtener inferencias debidamente fundamentadas utilizamos el método deductivo, el cual garantiza la corrección del razonamiento ya que se basa en la relación de consecuencia lógica que consiste en que de uno o varios enunciados iniciales (premisas) se obtiene otro (conclusión) con la condición de que si los primeros son verdaderos, el último necesariamente también lo será, es decir, es imposible que las premisas sean verdaderas y la conclusión falsa.

\section{ObJeto de ESTUdio de la ARChivística. Fondo Versus COlECción}

Independientemente de la respuesta que se proporcione a la discusión acerca de la cientificidad o no de la Archivística, cuestión que por demás excede los límites del presente trabajo, una constante que podemos descubrir como su objeto de atención es que siempre gira en torno al archivo. Según Tanodi (2009: 39. Resaltado propio), quien niega el carácter de ciencia a la Archivística, "se trata de una disciplina auxiliar o funcional de la administración y de la historia, que se refiere a la creación, historia, organización y funciones de los archivos". Quienes la consideran ciencia, que sin duda en la actualidad son mayoría, afirman que la Archivística es "la ciencia de los archivos" (Casanova, 1928: 24; Heredia, 1991: 29). Del mismo modo, el International Council on Archives (ICA, 1988: 20. Resaltado propio) indica que la Archivistique es la "Disciplina que trata los aspectos teóricos y prácticos de las funciones de los archivos", mientras que en inglés Archive(s) Administration se define como "El estudio teórico y práctico de políticas, procedimientos y problemas relacionados con las funciones del archivo".

La situación se complica cuando descubrimos que el término 'archivo' posee dos sentidos. Puede denotar tanto la institución que resguarda y gestiona los documentos archivísticos, como el conjunto de documentos con características específicas que los convierten propiamente en 'documentos archivísticos'. Entonces, el objeto de estudio puede ser la institución, los documentos archivísticos o ambos.

Antonia Heredia es muy clara al afirmar que la Archivística es "la ciencia de los archivos, no de los documentos” (1991: 29). Por el contrario, Roscoe R. Hill definió a la archivística "como una ciencia experimental encargada de la conservación y administración de los documentos" (en Ramírez Aceves (2018: 46)). Por su parte Cruz Mundet (1994: 64) indica que la Archivística es una ciencia emergente cuyo objeto son los archivos en su doble consideración: los fondos documentales y su entorno, es decir, la institución que los alberga. 
De lo anterior se desprende la importancia que toman los documentos archivísticos, ya sea como objetos directos de estudio o indirectamente, como componentes esenciales de la institución archivo. Por consiguiente, resulta esencial conocer sus características, las cuales fueron enunciadas desde el siglo XIX en el manual escrito por Muller, Feith y Fruin, considerado la obra fundacional de la Archivística como disciplina científica. Ya desde el inicio del primer capítulo del mencionado trabajo, "Origen y composición de los archivos”, introducen la definición que pasó a ser canónica para esa disciplina. Por archivo se entiende el "conjunto de documentos escritos, dibujos y material impreso, recibidos o producidos oficialmente por un órgano administrativo específico o por uno de sus funcionarios, en la medida en que tales documentos estaban destinados a permanecer bajo custodia de ese órgano o funcionario" (Muller, Feith y Fruin, 1973: 13. Resaltado propio). Más adelante, se hace explícita la consecuencia de esa relación entre actividad administrativa y los documentos que se producen en ese proceso: "Un archivo es un todo orgánico" (Muller, Feith y Fruin, 1973: 18).

Dicha idea de documento archivístico se ha repetido con algunos matices por autores posteriores, pero sin cambiar sus notas distintivas. Así, encontramos que Casanova (1928: 19) indica que "El archivo es la colección ordenada de los documentos de una entidad o individuo, constituido durante el desarrollo de su actividad y conservado para el logro de los fines políticos, jurídicos y culturales de esa entidad o individuo". Por su parte, Jenkinson (1937: 11. Resaltado propio) escribe que un documento es archivístico si fue elaborado o utilizado en el curso de una transacción administrativa o ejecutiva (ya sea pública o privada) de la cual formó parte; y posteriormente preservado junto con su información por la persona o personas responsable de esa transacción y sus legítimos sucesores. Heredia (1991: 89. Resaltado propio) afirma que

Archivo es uno o más conjuntos de documentos, sea cual sea su fecha, su forma y soporte material, acumulados en un proceso natural por una persona o institución pública o privada en e1 transcurso de su gestión, conservados, respetando aquel orden, para servir como testimonio e información para la persona o institución que los produce, para los ciudadanos o para servir de fuentes de historia.

El énfasis muestra la constante en las definiciones proporcionadas: el origen y acumulación de los documentos no son arbitrarios, son el resultado de un proceso "natural" de la actividad propia del ente generador -individuo o institución- lo que convierte al conjunto de dichos registros en un "todo orgánico natural", un sistema donde existen relaciones entre sus componentes y entre las mismas "relaciones que transcurren entre aquellos documentos" 
(Lodolini, 1991: 35). La naturalidad de su origen a partir de las actividades de la institución que crea éstas refleja la estructura, organización y funcionamiento de dicha institución. De este modo, llegamos al término fondo, que según el ICA (1988: 72) es "El cuerpo total de registros recopilados por una persona física o moral, institución u organización en particular en el ejercicio de sus actividades y funciones". La naturaleza de los fondos condiciona la metodología propia para su tratamiento en su organización, la cual, como ya es sabido, se rige por el principio de procedencia y orden originales.

Ahora bien, existe otra clase de conjuntos de documentos cuya naturaleza es totalmente opuesta al origen y acumulación natural de los documentos archivísticos. El origen de ese nuevo conjunto es artificial, la intencionalidad que guio la reunión de los documentos es subjetiva. A dicho conjunto de documentos se le denomina colección. Según el Diccionario de terminología archivística se entiende por tal "Una acumulación artificial de documentos de cualquier procedencia reunidos sobre la base de alguna característica común, por ejemplo, modo de adquisición, tema, idioma, medio, tipo de documento, recopilador". Y agrega: "Este término se opone al de fondo" (ICA, 1988: 40). Al tener un origen diferente al de los fondos, el tratamiento documentario no puede ser guiado por el mismo principio por el que se organizan éstos, por lo que "cuando hablamos de la organización de colecciones de documentos, estamos ante la posibilidad de aplicar métodos no necesariamente archivísticos" (Villanueva Bazán, 2000:59).

Ante esa oposición podemos encontrar incluso aseveraciones contundentes: "Quisiera que la palabra "colección" fuera suprimida del vocabulario del archivista" (Jenkinson, 1980: 238). Para Lodolini (1993: 269), los documentos que no son organizados siguiendo los métodos archivísticos "salen del campo de la archivística y entran en el de la ciencia de las informaciones". García Ruipérez también expresa su desacuerdo, ya que la aceptación de ese tipo de conjunto formado arbitrariamente de documentos "viene a 'normalizar' situaciones y actitudes hasta ahora cuanto menos cuestionables. Algunos consideramos que su inclusión es un paso atrás en el desarrollo de nuestra disciplina" (García Ruipérez, 2007: 13).

Sin embargo, a pesar de esa oposición teórica contra las colecciones, en la práctica los archivistas se tienen que enfrentar a ellas. En ocasiones, por lo general en archivos particulares, aunque también a veces en fondos institucionales, además de los fondos, existen colecciones. Las causas de la presencia de esos conjuntos documentales son variadas: desde una reunión azarosa de estampas, dibujos, fotografías (Heredia, 1991: 149) hasta partes inherentes de un expediente, como las fotografías de un proceso judicial o de una investigación empírico-experimental. También pueden ser restos de fondos de 
un archivo que han sido desintegrados del todo orgánico sin que sea posible recuperar la organicidad original. Asimismo, existen las llamadas secciones o series facticias que son "agrupaciones documentales realizadas voluntariamente en los archivos a partir de sus fondos y responden a motivos de conservación o instalación determinados [...] por sus soportes o por su grafía, aunque no han faltado [...] algunas razones subjetivas y arbitrarias [para] agrupar los documentos en razón de un tema [...]” (Heredia, 1991: 149).

Ante esa realidad es necesario dejar abierta la posibilidad para incluir a las colecciones dentro del campo de la Archivística. Así por ejemplo, Schellenberg escribe que esa ciencia "trata de los archivos, [...] de las colecciones de documentos que en los archivos se conservan como fuente para su conocimiento ulterior y servicio al público" (Schellenberg, 1987: 44. Resaltado propio). De la misma manera, Heredia reconoce que "Los documentos de archivo constituyen fondos documentales y excepcionalmente colecciones" (Heredia, 1991: 164. Resaltado propio).

Del lado de las bibliotecas que tienen fondos archivísticos también se percibe una preocupación, aunque no tanto a nivel epistemológico, sino práctico, debido a que el objeto de la Bibliotecología no se centró tanto en las colecciones, como la Archivística lo hizo con los fondos. Esos archivos en bibliotecas incluyen fondos personales, familiares, de empresas, instituciones, entre otros, y reúnen múltiples materiales, como fotografías, películas, dibujos y demás materiales gráficos; en soportes tradicionales analógicos o archivos digitales, etcétera; tienen incluso un papel clave en la investigación. Principalmente se encuentran en colecciones especiales y han sido reunidas poco a poco tanto por bibliotecas universitarias como públicas (Estivill Rius, 2008). El tratamiento documentario es diferente a lo practicado por los bibliotecarios, por lo que la preocupación es trabajarlos de manera adecuada para no destruir esos fondos con una metodología equivocada.

\section{COMPlementARIEDAD}

Ahora es necesario precisar el concepto de complementariedad tal como se maneja en el presente trabajo dentro de la teoría del concepto. Según el Diccionario de la lengua española (RAE, 2014), por complementariedad se entiende "Cualidad de complementario"; a su vez, complementario es un adjetivo "De complemento. Que sirve para completar o perfeccionar algo".

Para visualizar el significado de complementariedad según el diccionario de la RAE utilizaremos los diagramas de Euler-Venn. Representaremos el universo, simbolizado con la letra $\mathrm{U}$, mediante un rectángulo y otros conjuntos 
dentro de ese universo, simbolizados con letras mayúsculas $\mathcal{A}, \mathcal{B}, \mathcal{C}, \mathrm{D}, \mathrm{E}, \ldots$ con un círculo dentro de $\mathrm{U}$ :

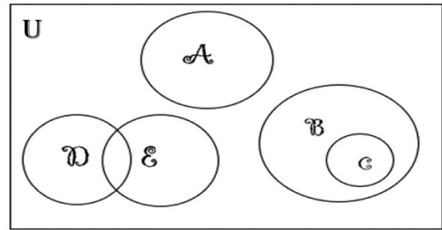

Figura 1

Fuente: elaboración propia

Como caso particular puede suceder que un conjunto $\mathcal{A}$ coincide con el universo, $\mathrm{U}=\mathcal{H}$ :

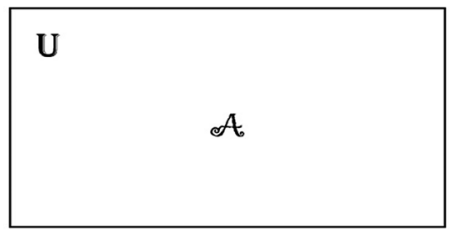

Figura 2

Fuente: elaboración propia

En un universo puede haber un conjunto A que tiene una forma "incompleta” y un conjunto B:

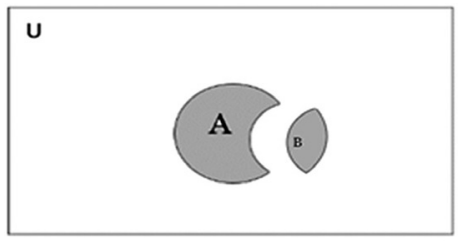

Figura 3

Fuente: elaboración propia

tales que si unimos A con B obtenemos un conjunto más completo: 


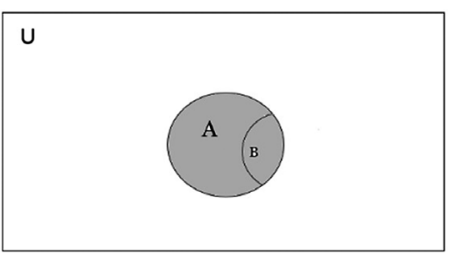

Figura 4

Fuente: elaboración propia

También puede darse el caso que el conjunto A casi coincide con el universo U a excepción de una zona vacía:

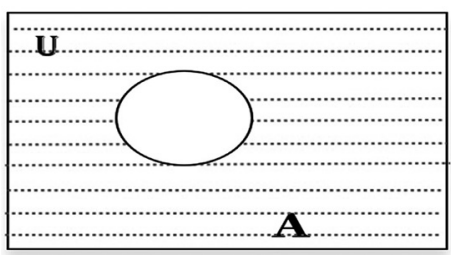

Figura 5

Fuente: elaboración propia

Debido a esa laguna, se dice que A está "incompleto". Para que abarque todo el universo $\mathrm{U}$ requiere de un conjunto $\mathrm{B}$

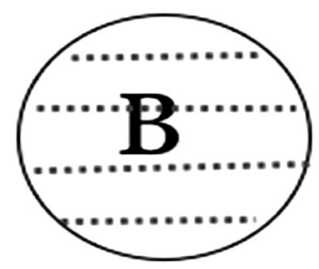

Figura 6

Fuente: elaboración propia

Así, A unión B da el universo:

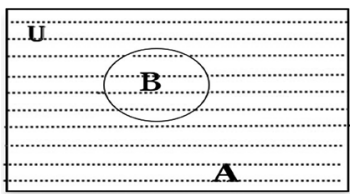

Figura 7 
Por consiguiente, es posible afirmar que B es complementario de A porque lo hace completo.

Sin embargo, ese no es el sentido cuando hablamos de complementariedad entre los documentos de un archivo y de una biblioteca. No es que un archivo está incompleto si no tiene colecciones, o una colección de biblioteca está incompleta si no tiene documentos de archivo, y se vuelven completos cuando los obtienen. Más aún, el hecho de que un archivo contenga colecciones ha sido fuertemente cuestionado y se valora en el mejor de los casos como excepción.

Cruz Mundet habla de otro tipo de complementariedad de las ciencias de la documentación (1994: 72-74), en donde el bibliotecario, el documentalista y el archivero "ya no están en conflicto, sino que son complementarios". Es un sentido cercano a la cooperación, a ofrecer y aprender de los otros. "Lo mismo que el médico, el dentista, el farmacéutico, el kinesiterapeuta no son rivales, sino que cooperan en beneficio de la salud pública. Al igual que los archiveros tienen aportaciones que hacer, tienen también mucho que aprender de los demás profesionales" (Cruz Mundet, 1994: 72). ${ }^{1}$

Ese tipo de cooperación entre las ciencias documentales, que para simplificar y en el contexto de este trabajo sólo ejemplificaremos con dos disciplinas: la Archivística y la Bibliotecología, se representa de la siguiente manera. Tenemos:

Un conjunto A que está incompleto para coincidir con el universo:

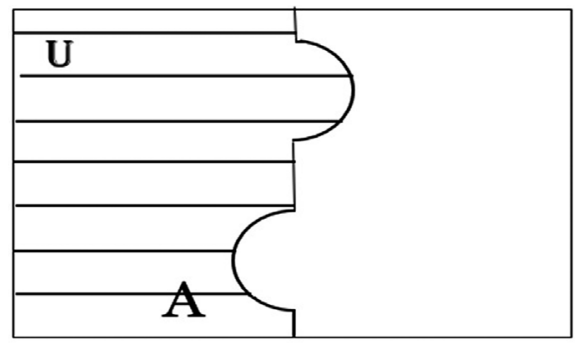

Figura 8

Fuente: elaboración propia

1 Ofrece un decálogo en el que concentra "puntos de encuentro, áreas comunes, retos similares, que aconsejan la confluencia y la colaboración más estrecha entre las tres ramas que componen las ciencias de la documentación, desde el respeto a la originalidad de cada cual" (Cruz Mundet, 1994: 74). Dichos puntos de encuentro son gestión de los documentos, nuevas tecnologías, soportes comunes, difusión de la información, lenguajes terminológicos, satisfacción de necesidades de información, acceso a la información, pobre base constitucional o jurídica, formación integrada y armónica de gestores de la información, y función informativa de la documentación (Cruz Mundet, 1994: 72-74). 
Un conjunto B que también está incompleto para coincidir con el universo:

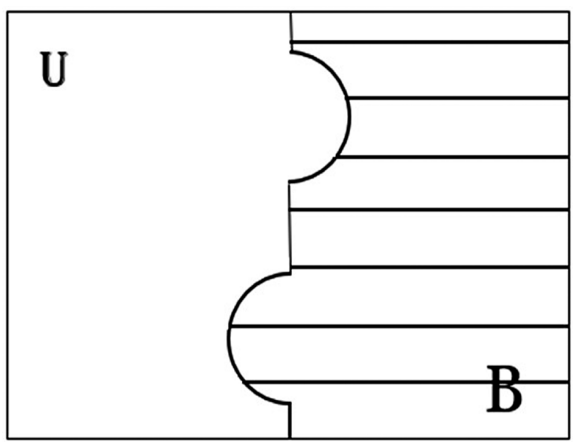

Figura 9

Fuente: elaboración propia

Pero entre A y B hay puntos de contacto, entradas y salientes que se complementan, lo que le falta a uno, lo tiene el otro y viceversa; por eso, cuando se unen, coinciden perfectamente y juntos conforman el universo:

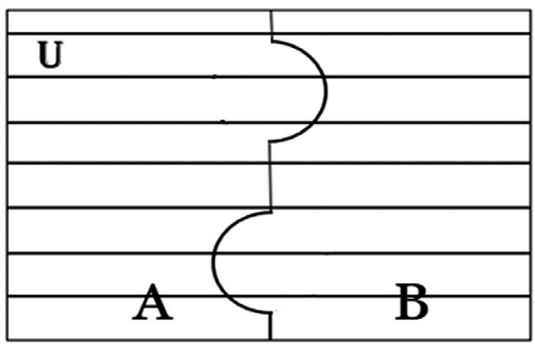

Figura 10

Fuente: elaboración propia

No obstante lo anterior, la idea de complementariedad es más compleja porque implica no sólo cooperar, aprender y enseñar, dar y recibir, sino una verdadera fusión en ciertos aspectos. El ejemplo que proporciona Cruz Mundet de la cooperación entre el médico, el dentista, el farmacéutico y el kinesiterapeuta nos ilustra la limitación de esa complementariedad, donde sólo se "ensamblan" sin tener un campo común a excepción del universo, en este caso la salud. No se le puede pedir a un médico que limpie una caries, a un dentista que realice una operación de apéndice, o a un kinesiterapeuta que practique una cesárea; pero el archivista sí debe organizar unas colecciones y el bibliotecólogo, unos fondos de archivo. Existe no sólo cooperación, sino compenetración en sus objetos de trabajo. Es por ello por lo que proponemos el concepto de complementariedad como se maneja en la teoría del concepto. 


\section{Complementariedad como relación entre conceptos}

Antes que nada, debemos precisar que por concepto se entiende una forma del pensamiento que identifica, distingue y agrupa a ciertos objetos con base en determinadas características (Voishvillo, 1989: 91). A estas últimas se les denominan contenido del concepto y a los conjuntos que se forman al agrupar los objetos que las poseen se les llama volumen del concepto. Para simplificar, simbolizaremos a los conceptos con letras mayúsculas y se sobreentenderá que esa misma letra denota su volumen.

Entre los conceptos se presentan algunas relaciones, pueden ser comparables y no comparables, compatibles e incompatibles, ser completos entre ellos (completud) o incompletos (incompletud). Si son compatibles, pueden estar entre sí como inclusión, equivalencia, subordinación, intersección o complementariedad. Por su parte, si son incompatibles, los conceptos pueden estar en co-subordinación o contradicción. Para fines de este trabajo, es necesario aclarar lo que es la compatibilidad, la completud y la inclusión entre conceptos para entender lo que es la complementariedad. ${ }^{2}$

\section{Definición 1. Compatibilidad-incompatibilidad}

Dos conceptos $\mathcal{A}$ y $\mathbb{B}$ son compatibles si y sólo si comparten por lo menos un elemento. Si no tienen ni un solo elemento común, son incompatibles.

\section{Definición 2. Completud-incompletud}

Dos conceptos $\mathcal{A}$ y $\$$ se encuentran en la relación de completud si y sólo si su unión da como resultado el universo. De lo contrario se encuentran en una relación de incompletud.

2 Las restantes relaciones entre conceptos son las siguientes:

Dos conceptos A y B son comparables si y sólo si coinciden sus universos. De lo contrario son incomparables.

Dos conceptos A y B son equivalentes si y sólo si son compatibles, el primero está incluido en el segundo, el segundo está incluido en el primero, y además están en relación de incompletud.

El concepto A se encuentra en la relación de subordinación con respecto al concepto B si y sólo si son compatibles, el primero está incluido en el segundo, pero no a la inversa, y están en relación de incompletud.

Dos conceptos A y B se encuentran en la relación de intersección si y sólo si son compatibles, el primero no está incluido en el segundo ni el segundo en el primero, y se encuentran en relación de incompletud.

Dos conceptos A y B se encuentran en la relación de co-subordinación si y sólo si son incompatibles, están en relación de incompletud.

Dos conceptos A y B encuentran en relación de contradicción si y sólo si son incompatibles y están en relación de completud.

Una explicación detallada de lo anterior se puede encontrar en Rendón Rojas (2017: 94-104). 
Definición 3. Inclusión-no inclusión

Un concepto $\mathcal{H}$ está incluido en el concepto $\mathbb{B}$ si y sólo si cada elemento de $\mathcal{A}$ es elemento de $\$$. Si existe por lo menos un elemento de $\mathcal{A}$ que no pertenece a B, A no está incluido en $B$.

\section{Definición 4. Complementariedad}

Dos conceptos $\mathcal{A}$ y $\mathbb{B}$ se encuentran en la relación de complementariedad si y sólo si: a) son compatibles, b) están en relación de completud, y c) no se incluyen mutuamente.

Por ejemplo, sea dentro del universo U de los números naturales,

A el concepto número menor de 1000, esto es, $\mathrm{A}=\{1,2,3, \ldots, 899,900,901,902, \ldots, 999\}$

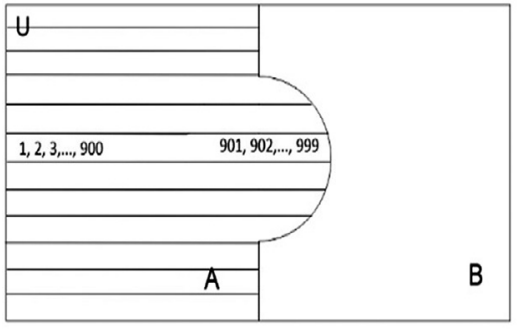

Figura 11

Fuente: elaboración propia

y B el concepto múmero mayor de 900, es decir, $\mathrm{B}=\{901,902,903, \ldots, 998,999,1000, \ldots\}$

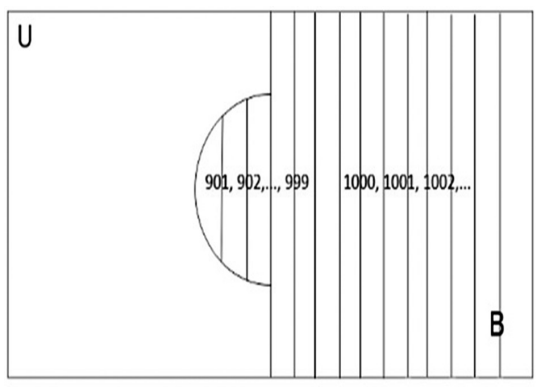

Figura 12

Fuente: elaboración propia 
La unión de A y B nos da como resultado:

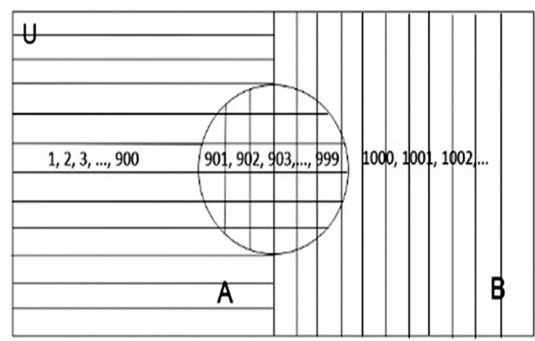

Figura 13

Fuente: elaboración propia

Este último conjunto cumple con las condiciones de a) compatibilidad: comparten por lo menos un elemento, los números del 901 al 999 son comunes a los dos conjuntos; b) completud: unidos conforman el universo de los números naturales; y c) no inclusión recíproca: existen elementos que sólo están en A, pero no en B: del 1 al 900; y otros que pertenecen a B, pero no contiene A: del 1000 al infinito. Por lo tanto, podemos concluir que A y B se encuentran en relación de complementariedad.

En el caso de los documentos de archivos y bibliotecas tenemos una situación idéntica. Si consideramos que el conjunto A es el conjunto de los documentos de los archivos, entonces tenemos que la gran mayoría son fondos archivísticos, pero en algunos podemos descubrir colecciones:

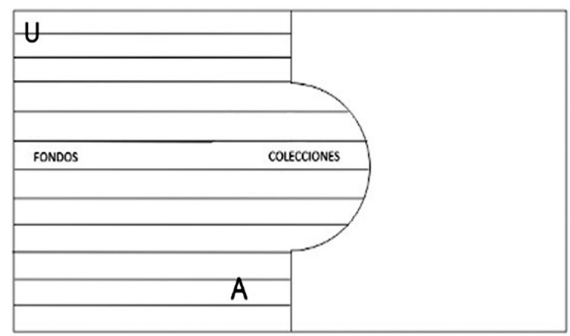

Figura 14

Fuente: elaboración propia

Al mismo tiempo, tenemos los documentos de las bibliotecas, los cuales como sabemos son colecciones, pero algunas albergan también fondos de algunos archivos: 


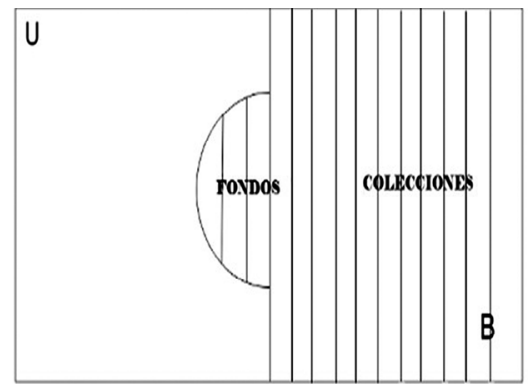

Figura 15

Fuente: elaboración propia

Si unimos los conjuntos A y B obtenemos el siguiente conjunto:

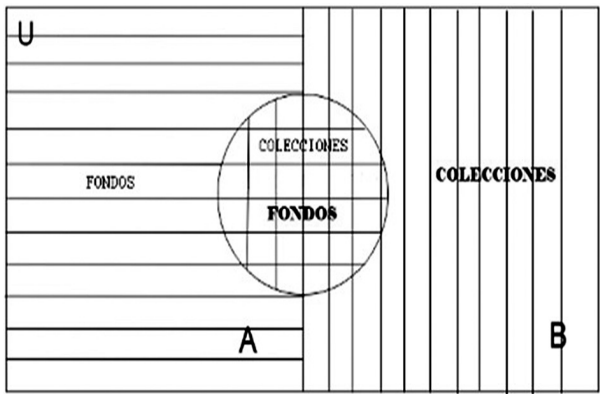

Figura 16

Fuente: elaboración propia

Observamos que se cumplen con las condiciones de a) compatibilidad: comparten por lo menos un elemento, en la zona cuadriculada; los dos comparten fondos y colecciones; b) completud: unidos conforman el universo de los documentos que satisfacen necesidades de información de usuarios en instituciones informativo-documentales, y c) no inclusión recíproca: existen elementos que sólo están en A, pero no en B (fondos sólo archivísticos que no están en bibliotecas) y otros que pertenecen a B, pero no contiene A (colecciones pertenecientes únicamente a bibliotecas, pero no a archivos). Por lo cual, de acuerdo con la definición de complementariedad, podemos inferir que entre A y B se presenta una relación de complementariedad, con lo cual se demuestra nuestra hipótesis principal. 


\section{Conclusiones}

Consideramos que con este análisis de la relación que guardan los conjuntos de documentos que albergan los archivos y las bibliotecas, utilizando un modelo teórico tomado de la teoría del concepto, hemos avanzado en la comprensión de dicha relación, alejándonos de la simple constatación del hecho y su aceptación como un caso anómalo. Sin embargo, es posible profundizar el conocimiento y el reto teórico que ahora se levanta es proporcionar las razones que determinan esa complementariedad. Esto nos conduce a cuestiones epistemológicas, la central de las cuales se refiere al problema del objeto de estudio de las disciplinas implicadas. Al solucionar dicho problema descubrimos que comparten elementos y podemos entender la naturaleza y mecanismo de la interdisciplina y transdisciplina entre esas ciencias. Simultáneamente, al realizar esas tareas tendremos que ampliar nuestra concepción del campo de la ciencia de la información documental si deseamos comprenderlo holísticamente, ya que se vislumbran relaciones de complementariedad, completud, intersección y convergencia entre otras disciplinas informativo-documentales como la Ciencia de la Información, la Documentación y la Museología, por lo que la inter- y transdisciplina se tendrán que expandir a ellas, todo lo cual valida nuestra hipótesis complementaria. Obviamente, la investigación a profundidad de esas cuestiones queda fuera de los objetivos del presente trabajo y merece un estudio posterior. Únicamente realizaremos un bosquejo de las problemáticas y posibles líneas que se pueden seguir.

Con respecto al objeto de estudio señalamos la necesidad de superar la idea de que la institución que alberga los acervos se convierta en objeto de estudio. ${ }^{3}$ La ciencia se hace sobre lo general y no lo individual, lo abstracto y no lo concreto. Los archivos y bibliotecas son organismos concretos y como tal, contingentes. Al respecto, Wersig (1992: 202) escribió: "Mientras no existan disciplinas como "ciencia del hospital" [hospital science] o "ciencia de la cárcel" [jailhouse science], algo así como "ciencia de la biblioteca" [library science] no es muy convincente". Peter van Mensch (1992) expresa la misma opinión para la Museología: así como no existe la 'escuela-logía' [school-ology] o iglesialogía [church-ology], tampoco puede existir la 'museo-logía [museum-ology].

Por lo tanto, para salir de la singularidad se deben buscar objetos de estudio más abstractos, como puede ser la información (Silva y Ribeiro, 2008; Thomassen, 2001), la información archivística (Fonseca, 2005), la información

3 En inglés es más evidente esta concepción desde el mismo nombre de las disciplinas: Archival Science y Library Science. 
orgánica (Couture, Ducharme y Rousseau, 1988) para la Archivística; el proceso de comunicación para la Documentación (López Yepes, 2015); el sistema informativo documental para la Bibliotecología (Rendón Rojas, 2005); lo museal para la Museología (Desvallées y Mairesse, 2010).

Al mismo tiempo, dentro de esas concepciones más abstractas de las disciplinas informativo-documentales, se reinterpretan las interrelaciones entre ellas; no sólo se perciben como puntos de contacto, colaboración, importación y exportación de conceptos, teorías, metodologías, temas, problemas, prácticas, sino como algo más orgánico donde la transversalidad está presente de manera permanente (Araújo, 2014), o donde comparten el núcleo duro de un Programa de Investigación Científica (Rendón Rojas, 2011), o donde convergen en la etapa postcustodial de la Ciencia de la Información para pasar de la interdisciplina a la transdisciplina (Silva y Ribeiro, 2008).

El camino para continuar la investigación en la epistemología de las disciplinas informativo-documentales está abierto, los puntos apenas mencionados son los siguientes problemas por analizar y con ello contribuir su desarrollo.

\section{REFERENCIAS}

Araújo, Carlos Alberto Ávila. 2014. Arquivologia, Biblioteconomia, Museologia e Ciência da Informação: O Diálogo Possivel. Brasília: Briquet De Lemos.

Casanova, Eugenio. 1928. Archivística. Siena: Lazzeri.

Couture, Carol, Jacques Ducharme y Jean-Yves Rousseau. 1988. "Larchivistique a-telle trouvé son identité?" Argus 17 (2): 51-60.

Cruz Mundet. 1994. Manual de Archivística. Madrid: Fundación Germán Sánchez Ruipérez; Pirámide.

Desvallées, André y François Mairesse. 2010. Conceptos claves de museología. París: Armand Colin, ICOM.

Estivill Rius, Assumpció. 2008. «Los fondos y las colecciones de archivo en las bibliotecas: modelos para su control y acceso». BiD: textos universitaris de biblioteconomia i documentació 21. https://doi.org/10.1344/105.000000327

Fonseca, Maria Odilia. 2005. Arquivologia e ciência da informação. Rio de Janeiro: FGV.

Gadamer, Hans-George. 1997. Verdad y método I. Fundamentos de una hermenéutica filosófica. Salamanca: Sígueme.

García Ruipérez, Mariano. 2007. "Series y tipos documentales. Modelos de análisis". Legajos. cuadernos de investigación archivística y gestión documental (10): 9-26. Fecha de consulta: 15 de octubre de 2020.

http://www.archiverosdiputaciones.com/wp-content/uploads/2015/07/ruiperez.pdf

Heredia, Antonia. 1991. Archivistica general. Teoría y práctica. 5a. ed. Sevilla: Diputación Provincial de Sevilla.

Hjørland, Birger. 2004. "Domain analysis: A Sociocognitive orientation for information science research". Bulletin of the American Society for Information Science and Tecbnology 30 (3): 17-21. https://doi.org/10.1002/bult.312 
ICA (International Council on Archives). 1988. Dictionary or Archival Terminology = Dictionnaire de Terminologie Archivistique: English and French with equivalents in Dutch, German, Italian, Russian and Spanish. Editado por Peter Walne. München; New York; London; Paris: K. G. Saur.

Jenkinson, Hilary. 1937. Manual of Archive Administration. London: Percy Lund, Humphries \& Co. LTD.

Jenkinson, Hilary. 1980. "The English Archivist: A new Profession. (An inaugural lecture for a new course in archive administration delivered at University College, London, October 14, 1947)", en Selected Writings of Sir Hilary Jenkinson, editado por Roger H. Ellis y Peter Walne Editors, 236-259. Reino Unido: Alan Sutton.

Kant, Inmanuel. 2007. Crítica de la razón pura. Buenos Aires: Colihue.

Lodolini, Elio. 1991. "El problema fundamental de la archivística: la naturaleza y el ordenamiento del archivo", en Archivística, coordinado por César Gutiérrez Muñoz, 30-51. Perú: Pontificia Universidad Católica del Perú.

Lodolini, Elio. 1993. Archivística. Problemas y principios. Madrid: Asociación Española de Archiveros, Bibliotecarios, Museólogos y Documentalistas.

López Yepes, José. 2015. La ciencia de la información documental: el documento, la disciplina y el profesional en la era digital. México: Universidad Panamericana.

Mensch, Peter van. 1992. "Towards a methodology of museology”. Tesis de Doctorado. Universidad de Zagreb, Croacia.

Muller, Samuel, Johan Feith y Robert Fruin. 1973. Manual de arranjo e descrição de arquivos. Rio de Janerio: Ministério de Justiça, Arquivo Nacional.

RAE (Real Academia Española). 2014. Diccionario de la lengua española, 23a. ed. Madrid: Espasa.

Ramírez Aceves, Merizanda María del Carmen. 2018. Elementos para fundamentar la cientificidad de la archivística. Toluca, Estado de México: Universidad Autónoma del Estado de México.

Rendón Rojas, Miguel Ángel. 2005. Bases teóricas y filosóficas de la bibliotecología. 2a. ed. México: UNAM-CUIB.

Rendón Rojas, Miguel Ángel. 2011. “A manera de conclusión”, en Bibliotecología, archivística, documentación: intradisciplina, interdisciplina o transdisciplina, coordinado por Miguel Ángel Rendón Rojas, 81-98. México: UNAM, Centro Universitario de Investigaciones Bibliotecológicas.

Rendón Rojas, Miguel Ángel. 2017. Introducción a la teoría de conjuntos, los operadores booleanos y la teoría del concepto para profesionales de la información documental. México: UNAM, Instituto de Investigaciones Bibliotecológicas y de la Información.

Schellenberg Theodore R. 1987. Archivos modernos. Principios y técnicas. México: Archivo General de la Nación.

Silva, Malheiro da Armando y Fernanda Ribeiro. 2008. Das ciências documentais à ciência da informação. Ensaio epistemológico para um novo modelo curricular, 2a. ed. Porto: Edições Afrontamento.

Tanodi, Aurelio. 2009. Manual de Archivología Hispanoamericana. Teoría y principios. Córdoba, Argentina: Editorial Brujas.

Thomassen, Theo. 2001. "A First Introduction to Archival Science". Archival Science 1,373-385.

Tognoli, Natália Bolfarini. 2010. "A contribuição epistemológica canadense para a construção da Arquivística Contemporânea”. Tesis de Mestrado em Ciência da Informação, Universidade Estadual Paulista, Faculdade de Filosofia e Ciências, Brasil. 
Torres Monroy, Luis. 2000. "Naturaleza de las colecciones documentales: el problema de su tratamiento archivístico", en Teoría y práctica archivística 1, 31-39. México: Coordinación de Humanidades, Centro de Estudios sobre la Universidad, Universidad Nacional Autónoma de México.

Villanueva Bazán, Gustavo. 2000. "La fotografía como documento de archivo y su procesamiento técnico”, en Teoria y práctica archivística 1, 57-67. México: Coordinación de Humanidades, Centro de Estudios sobre la Universidad, Universidad Nacional Autónoma de México.

Voishvillo, Evguenii Kasimírivich. 1989. Poniatie kak forma muishleniia. Lóguico-gnoseologuítchesii análiz [El concepto como forma del pensamiento]. Moscú: MGU.

Wersig, Gernot. 1992. "Information Science and theory: a weaver bird's perspective", en Conceptions of library and information science: Historical, empirical and theorical perspectives, editado por Pertti Vakkari y Blaise Cronin, 201-217. London: Graham Taylor.

Para citar este texto:

Rendón Rojas, Miguel Ángel. 2021. "Relación de complementariedad entre los documentos de los archivos y las bibliotecas”. Investigación Bibliotecológica: archivonomía, bibliotecología e información 35 (88): 63-82.

http://dx.doi.org/10.22201/iibi.24488321xe.2021.88.58372 